\title{
CORRECCIÓN ATMOSFÉRICA DE LAS IMÁGENES VISIBLES DEL SENSOR AVHRR - NOAA USANDO LOS MODELOS 5S Y SMAC
}

\author{
Joel Rojas Acuña ${ }^{\mathrm{a}}$, Arturo Lauro Gamarra ${ }^{\mathrm{a}}$, José Carlos Eche Llenque ${ }^{\mathrm{a}}$ \\ Carlos E. Quiche Surichaqui ${ }^{\mathrm{b}}$ \\ ${ }^{a}$ Laboratorio de Teledetección. Departamento de Física Interdisciplinaria. Facultad de Ciencias Físicas, \\ Universidad Nacional Mayor de San Marcos. Apartado postal 14 -0149, Lima 14 - Perú. \\ ${ }^{b}$ Laboratorio de Física. Universidad Nacional Agraria de la Selva. Av. Universitaria s/n Km. 1.5 - Tingo Maria - Perú.
}

\begin{abstract}
Resumen
En este trabajo se ha implementado el modelo de corrección atmosférica denominado "Simplified Method for the Atmospheric Correction" (SMAC). Este modelo consiste en determinar la relación entre la reflectancia estimada en la cima de la atmósfera y la reflectancia estimada en la superficie del suelo utilizando algoritmos matemáticos de transferencia radiativa, dispersión de Rayleigh, dispersión de aerosoles, etc. Se ha comparado los valores de la reflectancia en la cima de la atmósfera y la reflectancia de la superficie del suelo, obteniendo como resultado una relación lineal de la estas dos cantidades con un valor de la pendiente igual a 1.44. El valor de la pendiente mayor que 1 indica que se ha realizado una corrección atmosférica aceptable. Para validar el modelo se ha obtenido los valores de la reflectancia de la superficie del suelo utilizando el modelo $5 \mathrm{~S}$, obteniendo un coeficiente de correlación de 0.99 . Con esto se pretende ayudar a obtener parámetros geofísicos más exactos para los que se desea obtener a partir de imágenes de satélite.
\end{abstract}

Palabras claves: Corrección Atmosférica, Reflectancia, Teledetección, Atmósfera.

\begin{abstract}
In this work has been implemented the model of atmospheric correction denominated "Simplified Method for the Atmospheric Correction" (SMAC). It consists of determining the relation between the reflectance calculated on the top of the atmosphere and the reflectance calculated at the surface of the soil using mathematical algorithms of radiative transference, Rayleigh`s scattering, scattering of aerosols, and others. One has compared the values of reflectance in the top of the atmosphere and the reflectance of the surface of the ground, obtaining like result a linear relation of the these two amounts with a value of he slope equal to 1.44. The value of slope greater than 1 indicates that an acceptable atmospheric correction has been made. In order to validate the model has been obtained the values of the reflectance of the surface of the ground using the $5 \mathrm{~S}$ model, obtaining a coefficient of correlation 0.99. With this it is tried to help to obtain more exact geophysical parameters for those than it is desired to obtain from satellite images.
\end{abstract}

Keywords: Atmospheric correction, Reflectance, Teledetección, Atmosphere.

\section{Introducción}

En este trabajo se ha aplicado un modelo de corrección atmosférica para procesar las imágenes visibles (banda 1 y 2 ) del sensor AVHRR, del satélite NOAA. Se expone un método de corrección atmosférica adecuado a imágenes de satélite libres de nubes y que se encuentran en el rango del espectro visible $(0.58$ $-1.00 \mu \mathrm{m})$, con la finalidad de eliminar el efecto de los gases y aerosoles. Estos gases son los componentes principales que no permiten 
estimar con precisión algunos parámetros, tales como: la reflectancia de la superficie del suelo, Índice de Vegetación Normalizada (NDVI), la Temperatura de la Superficie del Suelo a partir de la radiancia detectada por el sensor AVHRR/NOAAA. Las imágenes de los satélites meteorológicos como el NOAA observan la tierra y miden la radiancia reflejada por el sistema Atmósfera - Superficie de la Tierra iluminada por el Sol. Esta señal depende de la superficie reflectora, que en su camino es perturbada por dos procesos atmosféricos, la absorción gaseosa y la dispersión por moléculas y aerosoles. La magnitud física recuperada de las imágenes es la reflectancia detectada por el sensor, o también conocida como la reflectancia en la cima de la atmósfera, la cuál se obtiene a partir de procesos de calibración de la imagen utilizando los niveles digitales de las imágenes y los parámetros de calibración. A partir de aquí se puede utilizar el método llamado SMAC (Simplified Method for the Atmospheric Correction) desarrollado por Rahman y Dedieu [1] para eliminar los efectos atmosféricos y obtener la reflectancia de la superficie del suelo. El algoritmo SMAC ha sido implementado como un modulo en el software de procesamiento de imágenes AVHHR / NOAA denominado "Pacha Ricaj" [2], para trabajar de forma semiautomática y asistida, requiriendo solamente de algunos parámetros de entrada. El modelo requiere para cada uno de los píxeles de la imagen los siguientes datos de entrada: el contendido de vapor de agua $\left(\mathrm{U}_{\mathrm{H} 2 \mathrm{O}}\right)$, contenido de ozono $\left(\mathrm{U}_{\mathrm{O} 3}\right)$, profundidad óptica a $550 \mathrm{~nm}$. $\left(\tau_{550}\right)$, los ángulos de observación, presión y la reflectancia en la cima de la atmósfera. En nuestro modelo se ha asumido que los parámetros $\tau_{550}, \mathrm{U}_{\mathrm{H} 2 \mathrm{O}}, \mathrm{U}_{\mathrm{O} 3}$, son los mismos para todos los píxeles. Los resultados de la reflectancia de la superficie del suelo obtenido utilizando el modelo SMAC han sido comparados con los resultados obtenidos por otro modelo de transferencia radiativa, llamado $5 \mathrm{~S}$ (Simulation of the Satellite Signal in the Solar Spectrum). Además se ha comparado la reflectancia en la cima de la atmósfera y la reflectancia de la superficie del suelo con SMAC para evaluar el efecto de la atmósfera en la señal recibida por el sensor AVHRR. La contribución de la atmósfera se ha estimado en un valor promedio de $11.13 \%$ de la señal recibida por el sensor AVHRR/NOAA

\section{Fundamentos Físicos}

\subsection{Descripción de las Funciones Analíticas para el Modelo "SMAC"}

Para describir la interacción de la radiación solar con la atmósfera necesitamos conocer la profundidad óptica, el albedo de dispersión simple de la atmósfera, el factor de asimetría, y las funciones de fase de dispersión [1].

\subsection{Dos Formas de la Transmisión Gaseosa}

El doble camino de la transmitancia gaseosa $t_{g i}$ para un gas dado es una función de la cantidad de absorbedores y las masas de aire. La ecuación es similar a la ley de transmisión de Bouguer y puede ser escrito como:

$$
t_{g_{i}}\left(\theta_{s}, \theta_{v}\right)=e^{\left(a(m U)^{n}\right)}
$$

Donde $m$ es la masa de aire dada por la expresión

$$
m=\frac{l}{\operatorname{Cos}\left(\theta_{s}\right)}+\frac{l}{\operatorname{Cos}\left(\theta_{v}\right)}=\frac{l}{\mu_{s}}+\frac{l}{\mu_{v}}
$$

y $U$ es la cantidad de absorbedores integrada verticalmente. Para una banda espectral dada, $a$ y $n$, son constantes y son ajustadas a los resultados del modelo 5S para cada uno de los gases separadamente. Los coeficientes $a$ y $n$ varían con la banda espectral. Para varios gases, la transmitancia es obtenida multiplicando cada una de las transmisiones individuales:

$$
\operatorname{tg}=\prod_{l}^{n} t_{g i} .
$$

\subsection{Transmisión Atmosférica Total $($ directa + difusa $)$}

Usando una aproximación empírica para la transmisión difusa total y expresamos como una función del espesor óptico de dispersión de aerosoles total a $550 \mathrm{~nm}$ y de los ángulos solares y de observación: es dado por 


$$
T(\theta)=a_{0}+\frac{a_{1} \tau_{550}}{\cos (\theta)}+\frac{a_{2}}{(l+\cos (\theta))} .
$$

Donde $a_{0}, a_{1}, a_{2}$ son tres constantes a ser ajustadas para una banda espectral y un tipo de aerosol. Esta transmisión toma en cuenta ambos casos, la dispersión de Rayleigh y aerosoles. Cuando $\tau_{550}=0$, la transmisión corresponde a la dispersión de Rayleigh, pero, en este caso, la exactitud depende del rango de profundidades ópticas de aerosoles usadas para ajustar los coeficientes de dicha ecuación. La absorción de aerosoles, si existe, es implícitamente tomada en cuenta en el coeficiente $a_{1}$, dependiendo del modelo de aerosol. Para tratar los efectos del entorno, la transmitancia difusa es calculada por:

$$
t_{d}(\theta)=T(\theta)-e^{-\tau / \mu}
$$

Donde $\tau$ es la profundidad óptica promediada para dispersión molecular y para dispersión y absorción de aerosoles.

\subsection{Dispersión de Rayleigh.}

Para una atmósfera molecular, la dispersión de primer orden da la reflectancia atmosférica de Rayleigh fue obtenida del modelo 5S:

$$
\rho_{\text {ar }}=\frac{\tau_{r} p_{r}(\xi)}{4 \mu_{s} \mu_{v}}
$$

Donde $\quad p_{r} \square(\xi)$ es la función de fase de dispersión molecular. La función de fase especifica la dispersión angular de luz por la atmósfera que da la probabilidad diferencial de la radiación dispersada en una dirección dada. El espesor óptico molecular, $\tau_{r}$, es una constante para una banda espectral dada y puede ser directamente tabulada a partir de los resultados del 5S. Una corrección para la variación de la presión debe ser introducida por,

$$
\tau_{r}(P)=\tau_{r}\left(P_{0}\right) \frac{P}{P_{0}} .
$$

Donde $\tau_{r}\left(P_{0}\right)$ es la profundidad óptica molecular a algún nivel de referencia $\left(P_{0}=1013.25 \mathrm{hpa}\right.$ para condiciones estándar) y $P$ es la presión observada [3]. De acuerdo a Tanre [4], la función de fase de dispersión molecular es aproximada por la siguiente ec (8):

$$
P_{r}(\xi)=1.5 \frac{(1-\delta)}{2+\delta}\left(1+\operatorname{Cos}^{2}(\xi)\right)+3 \frac{\delta}{2+\delta}
$$

Donde $\delta$ es el factor molecular de polarización ( $\delta=0.0139)$, y $\xi$ es el ángulo de dispersión calculado a partir de la siguiente ecuación:

$\cos (\xi)=-\left[\mu_{s} \cdot \mu_{v}+\sqrt{\left(1-\mu_{s}^{2}\right)\left(1-\mu_{v}^{2}\right)} \cos (\Delta \Phi)\right]$

\subsection{Dispersión de Aerosoles}

La reflectancia atmosférica por aerosoles sobre una banda espectral es calculada a partir de la siguiente ec (10).

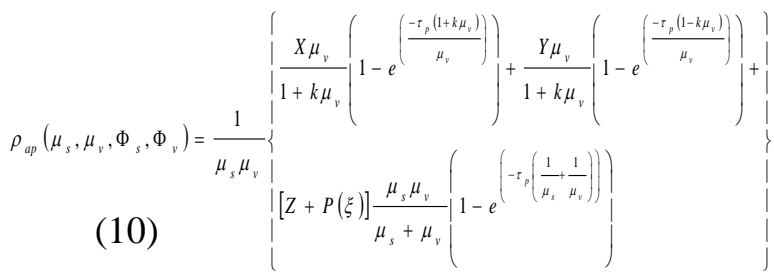

Con $k^{2}=\left(1-\omega_{0}\right)\left(3-\omega_{0} \beta_{1}\right)$ donde $\beta_{1}=3 g, \mathrm{~g}$ es el factor asimétrico y $\omega_{0}$ es el albedo de dispersión simple de la atmósfera. La profundidad óptica de aerosol promedio para una banda espectral dada $\tau_{p}$, es calculada a partir de la profundidad óptica dada a $550 \mathrm{~nm}$ usando una ecuación lineal como sigue:

$$
\tau_{\mathrm{p}}=\mathrm{a}_{0}+\mathrm{a}_{1} \tau_{550}
$$

Donde $a_{0}$ y $a_{l}$ son los coeficientes a ser ajustadas para una banda espectral dada. La función de fase de aerosol es obtenido a partir de una ecuación polinomial de segundo orden del ángulo de fase, $\xi$, en grados:

$$
p_{p}(\xi)=a_{0}+a_{1} \xi+a_{2} \xi^{2}
$$

Donde $a_{0}, a_{1}, a_{2}$, son coeficientes a ser ajustada para una banda espectral dada. La reflectancia atmosférica total es finalmente dada por una simple suma de los dos términos:

$$
\rho_{a}=\rho_{a r}+\rho_{a p}
$$




\section{Datos y Procesamiento}

Los datos imágenes usados en el presente trabajo han sido obtenidos del archivo activo de la NOAA que se encuentran disponibles en "The Comprehensive Large Array-data Stewardship System" (CLASS). Esta es una biblioteca electrónica de datos medioambientales de la NOAA. La página Web es http://www.class.noaa.gov. Se han seleccionado las imágenes LAC (Cobertura de Área Local) en formato Level-1b con una resolución espacial de $1.1 \mathrm{~km}$. en el nadir.

La Figura 1 muestra una composición en falso color de las bandas 1, 2 y 5 del sensor AVHRR/3 del satélite NOAA-16. Las nubes presentes en estas imágenes son un problema serio, pues no permite recuperar la reflectancia de la superficie del suelo. Los lugares cubiertos por nubes deben ser filtrados antes de aplicar el modelo de corrección atmosférica SMAC.
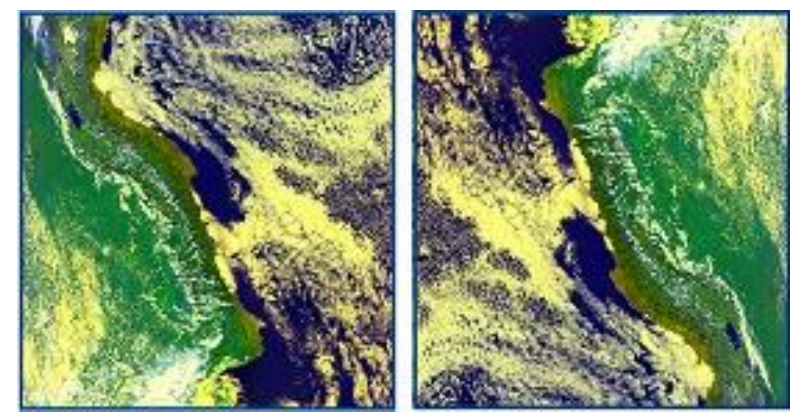

Fig. 1. Imagen del sensor AVHRR/3 del satélite de orbita polar NOAA-16. Es una composición en falso color (R:1, G:2, B:5).

Los algoritmos para realizar la corrección atmosférica de las imágenes AVHRR/NOAA se han implementado en el software de procesamiento de imágenes AVHRR/NOAA Pacha Ricaj, diseñado en el entorno de programación Builder $\mathrm{C}++$. En tanto que para el proceso se utilizado los siguientes tablas 1, 2,3 y 4. Estos coeficientes se ingresan en el programa con una extensión *.DAT y está dado para cada tipo de atmósfera, en nuestro caso hemos considerado los coeficientes para el tipo continental, es por eso que se ingresa este archivo con la siguiente denominación: "coef_NOAA16VIS_CONT.dat"

\begin{tabular}{|c|c|c|}
\hline \multicolumn{3}{|c|}{$\begin{array}{l}\text { Tabla 1. Coeficientes para calcular la transmisividad } \\
\text { vapor de agua para diferentes bandas espectrales } \\
\qquad g_{\mathrm{H}_{2} \mathrm{O}}\left(\theta_{s}, \theta_{v}\right)=\operatorname{Exp}\left(a\left(m U_{\mathrm{H}_{2} \mathrm{O}}\right)^{n}\right)\end{array}$} \\
\hline Banda espectral & A & $\mathrm{n}$ \\
\hline AVHRR/3 NOAA-16 & -0.004506 & 0.764571 \\
\hline
\end{tabular}

\begin{tabular}{|c|c|c|c|}
\hline \multicolumn{4}{|c|}{$\begin{array}{l}\text { Tabla 2. Coeficientes para calcular la transmisividad del } \\
\text { Oxigeno } \\
\qquad g_{O_{2}}\left(\theta_{s}, \theta_{v}\right)=\operatorname{Exp}\left(a(m)^{n}\right) U_{o_{2}}=1\end{array}$} \\
\hline Banda espectral & A & $\mathrm{n}$ & $\mathbf{P}$ \\
\hline AVHRR/3 - NOAA-16 & -0.000718 & 0.593404 & 1.646391 \\
\hline
\end{tabular}

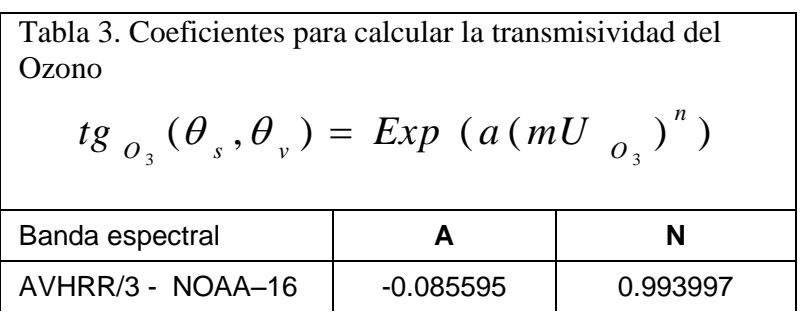

Tabla 4. Profundidad óptica de Rayleigh $\tau_{r}$, y Albedo esférico $S_{r}$, Para atmósferas puramente de Rayleigh (Solamente moléculas no aerosoles)

\begin{tabular}{|l|c|c|}
\hline Banda espectral & $\tau_{r}$ & $S_{r}$ \\
\hline AVHRR/3 - NOAA-16 & 0.056574 & 0.050341 \\
\hline
\end{tabular}

\section{Resultados y Discusión}

La imagen que se muestra en la Figura 2, corresponde a una imagen de la banda 3 (vapor de agua, $1.580-1.64 \mu \mathrm{m}$ ) captada por el sensor AVHRR/3. Es una imagen en formato original, es decir, se muestra en niveles de gris con el tipo de dato entero, tal como nos lo envía el CLASS. (No se ha hecho ningún tipo de corrección), y es visualizada por el Software Pacha Ricaj. El Software "Pacha Ricaj" tiene la capacidad de realizar la corrección atmosférica, utilizando el código SMAC diseñado por Rhaman y Dediu [1], solamente ingresando algunos parámetros de entrada. Los valores utilizados para el contendido de vapor de agua $\left(\mathrm{U}_{\mathrm{H} 2 \mathrm{O}}\right)$, contenido 
de ozono $\left(\mathrm{U}_{\mathrm{O} 3}\right)$, profundidad óptica a $550 \mathrm{~nm}$. $\left(\tau_{550}\right)$ y la presión son los que se muestran en la Figura 3.

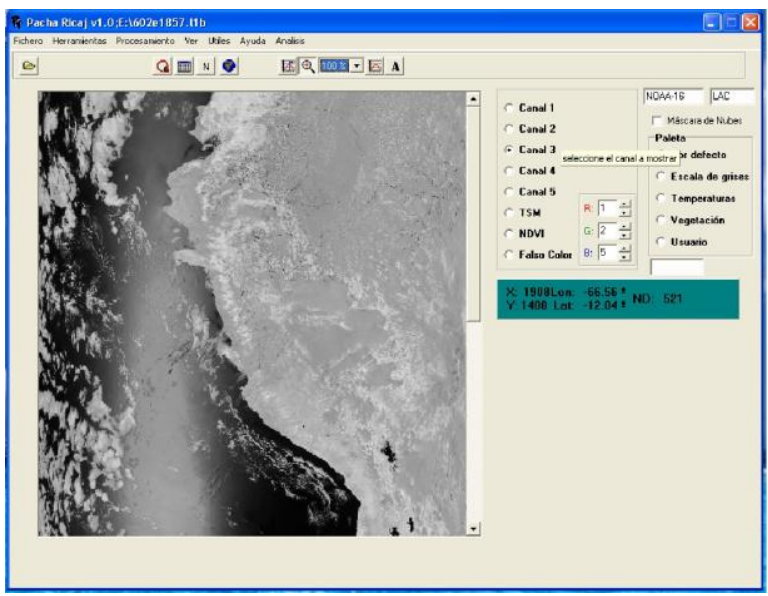

Fig.2. Ventana principal del software de procesamiento de imágenes AVHRR/NOAA, Pacha Ricaj. Se muestra la imagen original del sensor AVHRR- Banda 3

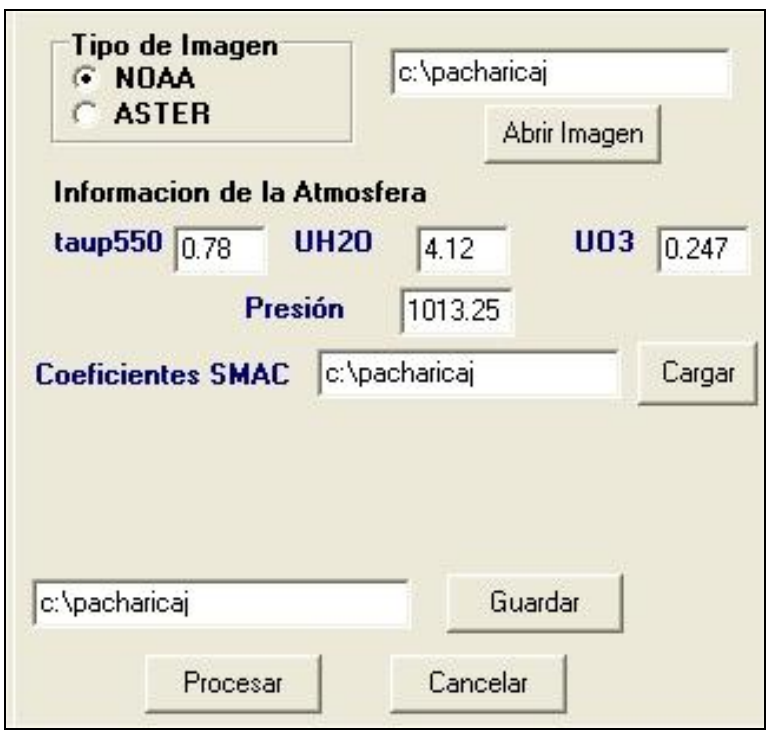

Fig. 3. Valores de los Parámetros de entrada del modelo SMAC

En la Figura 4 se muestra los la imagen AVHRR/NOAA antes de corregirla atmosféricamente (Imagen izquierda) y la misma imagen corregida atmosféricamente (imagen derecha). El modulo de corrección atmosférica implementado en el software "Pacha Ricaj" permite comparar los resultados obtenidos sobre una imagen antes y después de realizar la corrección atmosférica.

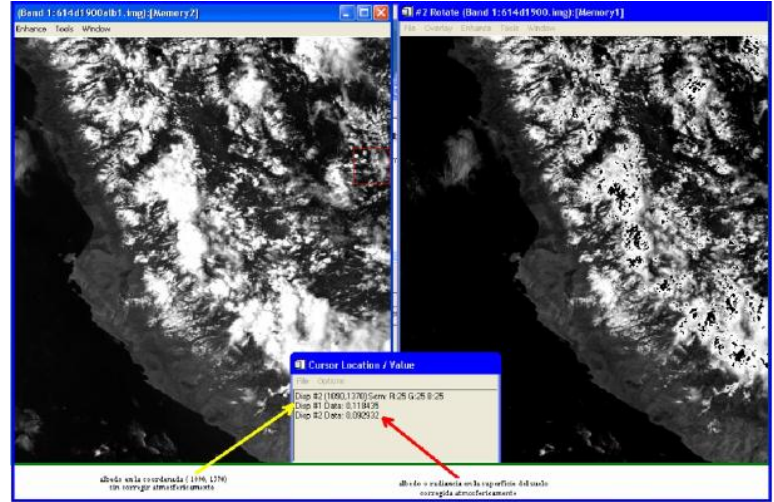

Fig. 4. Comparación entre la imagen antes de ser corregida atmosféricamente y corregida atmosféricamente, en el cuadro pequeño se observa los valores para un determinado píxel.

En la Tabla 5 se muestran los resultados obtenidos de la reflectancia de la superficie del suelo utilizando los modelos 5S y SMAC para cada uno de los píxel indicados. El coeficiente de Correlación de estos datos es 0.99 y se muestra en la Figura 5.

Tabla 5

$\begin{array}{cccc}\text { COOR(X) } & \text { COOR(Y) } & \text { 5S } & \text { SMAC } \\ 1580 & 641 & 0.224081 & 0.235284 \\ 1929 & 557 & 0.56578 & 0.871216 \\ 1575 & 519 & 0.149815 & 0.108049 \\ 1565 & 626 & 0.228265 & 0.243187 \\ 1640 & 673 & 0.127849 & 0.061831 \\ 1572 & 587 & 0.227742 & 0.241943 \\ 1942 & 641 & 0.56578 & 0.882628 \\ 1642 & 739 & 0.139355 & 0.081387 \\ 1736 & 744 & 0.127849 & 0.047171 \\ 1709 & 679 & 0.174396 & 0.137419 \\ 1740 & 794 & 0.171258 & 0.128584 \\ 1640 & 460 & 0.107452 & 0.02522 \\ 1620 & 635 & 0.185379 & 0.166377 \\ 1665 & 840 & 0.188517 & 0.166792 \\ 1776 & 825 & 0.180149 & 0.14213 \\ 1668 & 850 & 0.188517 & 0.166546 \\ 1808 & 1543 & 0.238202 & 0.247582 \\ 1828 & 1587 & 0.205776 & 0.186286 \\ 1780 & 1468 & 0.169689 & 0.120301 \\ 1576 & 593 & 0.26782 & 0.307911 \\ 1586 & 664 & 0.39006 & 0.500827 \\ 1539 & 613 & 0.151384 & 0.114943 \\ 1545 & 611 & 0.145108 & 0.103567 \\ 1560 & 621 & 0.221989 & 0.233056 \\ 1629 & 687 & 0.135171 & 0.076398\end{array}$




$\begin{array}{cccc}1743 & 727 & 0.124188 & 0.0388991 \\ 1729 & 799 & 0.553556 & 0.759975 \\ 1635 & 561 & 0.103268 & 0.017433 \\ 314 & 3626 & 0.052014 & 0 \\ 448 & 3419 & 0.529108 & 0.714887 \\ 1786 & 1459 & 0.201069 & 0.178096 \\ 1789 & 1357 & 0.646764 & 0.912726 \\ 1780 & 1471 & 0.177534 & 0.13513 \\ 1802 & 1357 & 0.623844 & 0.881207 \\ 1709 & 1270 & 0.048876 & 0 \\ 1541 & 1361 & 0.52758 & 0.692024 \\ 1755 & 1299 & 0.151907 & 0.08925 \\ 1760 & 1232 & 0.153476 & 0.092635 \\ 1741 & 1201 & 0.203161 & 0.186389\end{array}$

Resultados 5S y SMAC

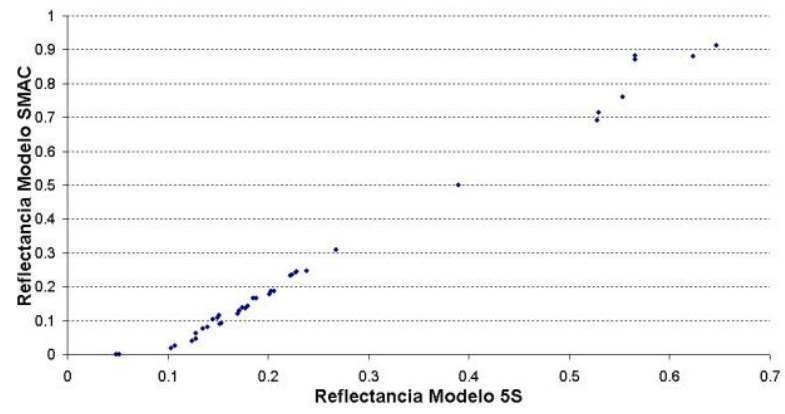

Fig. 5. Reflectancia de la superficie del suelo obtenidos por los modelos 5S y SMAC.

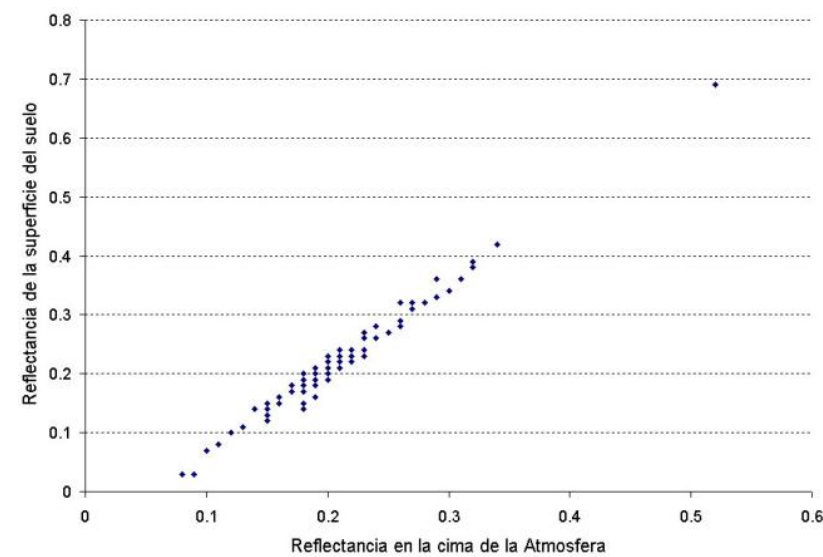

Fig. 6. Gráfica de las reflectancias calculada en la cima de la atmósfera y la reflectancia de la superficie del suelo. La pendiente de esta distribución es 1.44.

En la Figura 6 se muestra la relación entre la reflectancia en la cima de la atmósfera (sin corrección atmosférica) y la reflectancia de la superficie del suelo (Corregido atmosférica).

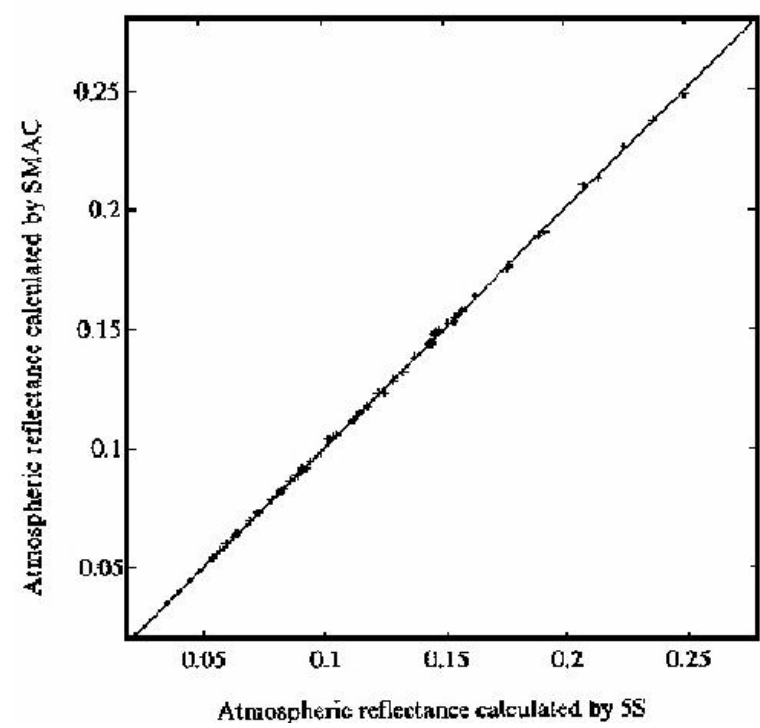

Fig. 7. Reflectancia atmosférica calculada por el SMAC y 5S según TANRE y DEDIU.

Se observa que la distribución es lineal con un valor de la pendiente iguala 1.44. Un valor mayor que 1 indica que se ha realizado una corrección atmosférica aceptable, es decir se ha eliminado el efecto de la atmósfera. Según estos resultados la contribución de la atmósfera promedio $11.13 \%$. Estos resultados deben ser tomados cuidadosamente pues deben ser validados con medida in situ para su fortalecimiento. En la Figura 7 se muestra el resultado obtenido por el trabajo realizado por Rhaman y Dediu [1].

\section{Conclusiones}

Los resultados presentados en este trabajo muestran un metodo simple de correccion atmosferica comparado con el 5S, para el calculo de procesos atmosfericos en la cima de la atmosfera. El modelo "SMAC" esta siendo usado para estudios de una cierta composicion de ${ }^{6} 6$ aproxiamadamente un año de imágenes con una rapidez de 30 segundos en una PC, pero sin embargo tiene ciertas restriciones, pues su equivalencia con otros modelos todavia carece de una calculo de modelo tranferencia radiativa exacta. Usando el metodo SMAC se puede reducir el tiempo de procesamiento para imágenes de satelite, pues reduce la carga de procesamiento en las computadoras. En el caso de estaciones receptoras de imágenes sepuede usar este metodo en la correccion atmosferica y 
dar resultados casi en tiepo real, y para usuarios que trabajan con gran cantidad de datos historicos.

Los coeficientes dados en este trabajo fueron calculados usando el codigo $5 \mathrm{~S}$ para un modelo de aerosol continental, pues este metodo permite tambien usar otros codigos de transferencia radiativa y puede ser extendido para icorporar otros modelos de aerosol.

\section{AGRADECIMIENTOS.}

Un agradecimiento especial a todos los miembros del Laboratorio de Teledetección de la Facultad de Ciencias Físicas. Este trabajo es parte de los proyectos que se están desarrollando cada año y que son financiados por el Consejo Superior de Investigaciones. Parte de este artículo condujo a la monografía de la licenciatura en Física de uno de los autores (ALG) en el año 2005 [5]. Agradecemos también al CONCYTEC por los proyectos financiados en el 2000 y 2001 y que sin ello no seria posible este trabajo.

\section{Referencias}

[1] Rahman, H. and G, Dediu. SMAC: a simplified method for the atmospheric correction of satellite measurements in the solar spectrum.

[2] Pariona, E., C. Eche, J. Martín, F. Carrillo y J. Rojas. (2002): Software de Procesamiento de Imágenes del Sensor AVHRR / NOAA. Revista de Investigación de la Electrónica. UNMSM. $\mathrm{N}^{\circ} 8$, Marzo.

[3] Teillet, P.M. (1990): Rayleigh optical depth comparisons from various sources: Applied Optics, 28, 1897-1900.

[4] Tanré et al. (1990): Description of a computer code to simulate the satellite signal in the solar spectrum: The 5S code. In: Int. J. Remote Sens., 11(4): 659-668

[5] Lauro, A. (2005): Corrección atmosférica de las imágenes en el espectro visible del satélite NOAA-16. Monografía de la Tesis de Licenciatura en Física. Facultad de Ciencias Físicas. Universidad Nacional Mayor de San Marcos. 